\title{
Antiproton Noise Source for the Tevatron
}

\author{
D. McConnell and B. Fellenz \\ Fermi National Accelerator Laboratory \\ P.O. Box 500, Batavia, Illinois 60510
}

October 1992 


\section{Disclaimer}

This report was prepared as an account of work sponsored by an agency of the United States Government. Neither the United States Government nor any agency thereof, nor any of their employees, makes any warranty, express or implied, or assumes any legal liability or responsibility for the accuracy, completeness, or usefulness of any information, apparatus, product, or process disclosed, or represents that its use would not infringe privately owned rights. Reference herein to any specific commercial product, process, or service by trade name, trademark, manufacturer, or otherwise, does not necessarily constitute or imply its endorsement, recommendation, or favoring by the United States Government or any agency thereof. The views and opinions of authors expressed herein do not necessarily state or reflect those of the United States Government or any agency thereof. 


\section{Antiproton Noise Source for the Tevatron \\ D. McConnell and B. Fellenz}

\section{October 1992}

\section{Introduction}

A new system for exciting the beam in the Tevatron has been installed in the A1 service building and in the A17 medium straight section. The purpose of the system is to make betatron tune measurements.

\section{Block Diagram}

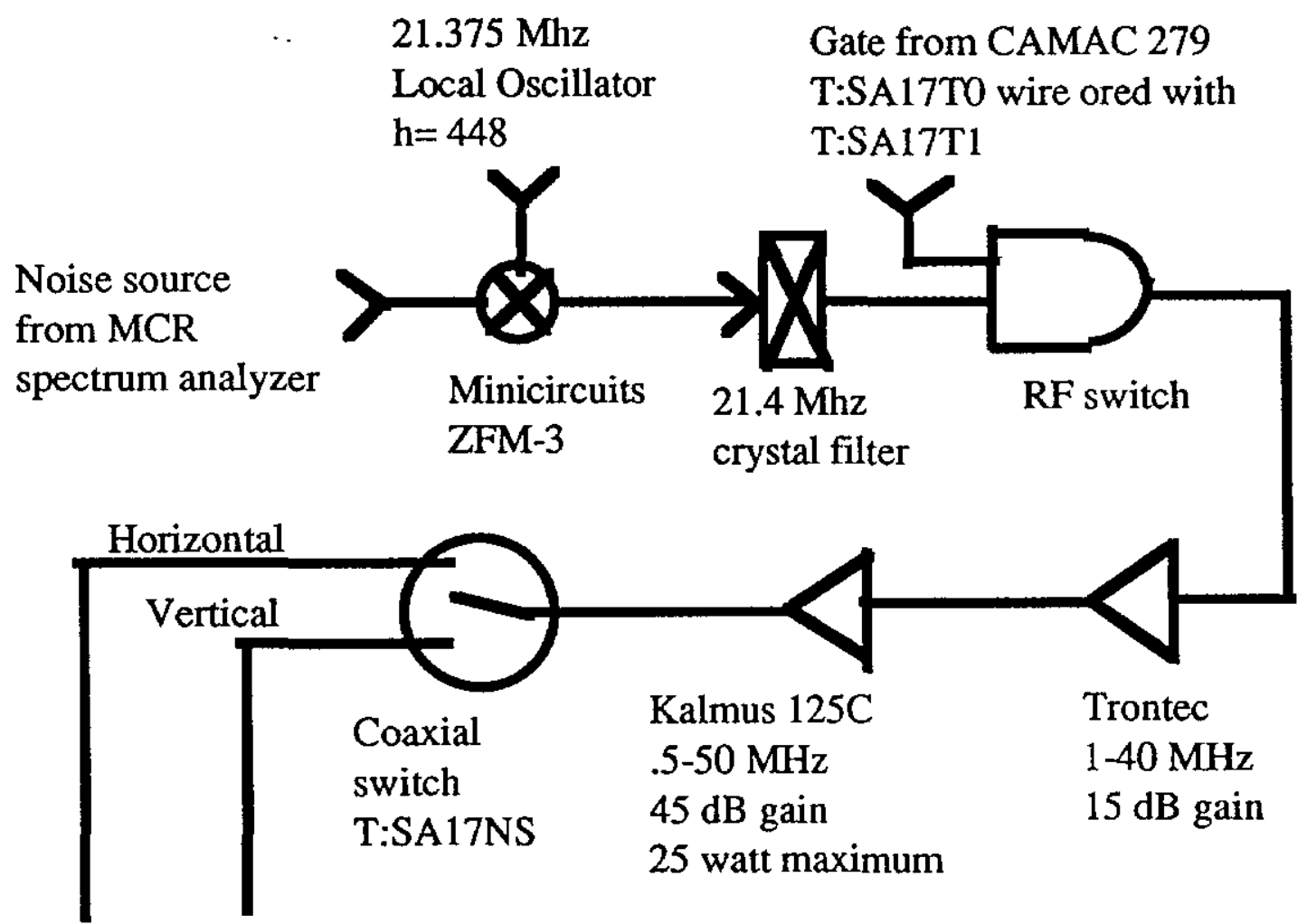

Cables to tunnel Anzac H-1-4 180 hybrids, Tevatron BPMs and back upstairs to 50 watt terminations.

\section{Description of operation}

The tune excitation system has been configured so that the attenuator in the Spectrum analyzer provides $40 \mathrm{~dB}$ of nearly linear excitation range. When the noise source is turned off, there is an 
additional $30 \mathrm{~dB}$ decrease in the noise level at the output of the 25 watt power amplifier. Thus, the system does produce unwanted beam emittance growth when it is turned off. The timing channels can be disabled to further increase the isolation when the system is off. To excite antiprotons after cogging has occurred, timing channel T:SA17T0 is set to $154 \mathrm{RF}$ cycles and timing channel T:SA17T0 is set to $336 \mathrm{RF}$ cycles. Both channels are triggered on teavatron beam sync clock events \$AA, \$BB, and \$CC. Channel 1 should always be delayed from channel 0 by $182 \mathrm{RF}$ cycles in order to produce six gate pulses for the six antiproton bunches per turn. Coaxial switch T:SA17NS is used to direct the noise to either the horizontal or the vertical standard tevatron BPM plates. At present horizontal is coded as "positive" in the parameter page basic control and status functions, and vertical is coded as "negative".

\section{Results of initial testing}

The tune excitation system is capable of selectively exciting the antiproton schottky signals by $14 \mathrm{db}$. (see attached spectrum analyzer plots) The full power emittance growth rate is measured to be .71 pi-mm-mrad per 5 minutes. LOSTP and LOSTPB were quiet during the excitation.

Time of wire fly

10:46 pm (before studies)

$11: 16 \mathrm{pm}$

$11: 31 \mathrm{pm}$ (before $5 \mathrm{~min}$ full on)

11:50 pm (after 5 min full on)

12:20 am vertical emittance pi-mm-mrad 18.1776 20.4711

18.4009

20.3352

19.9603 

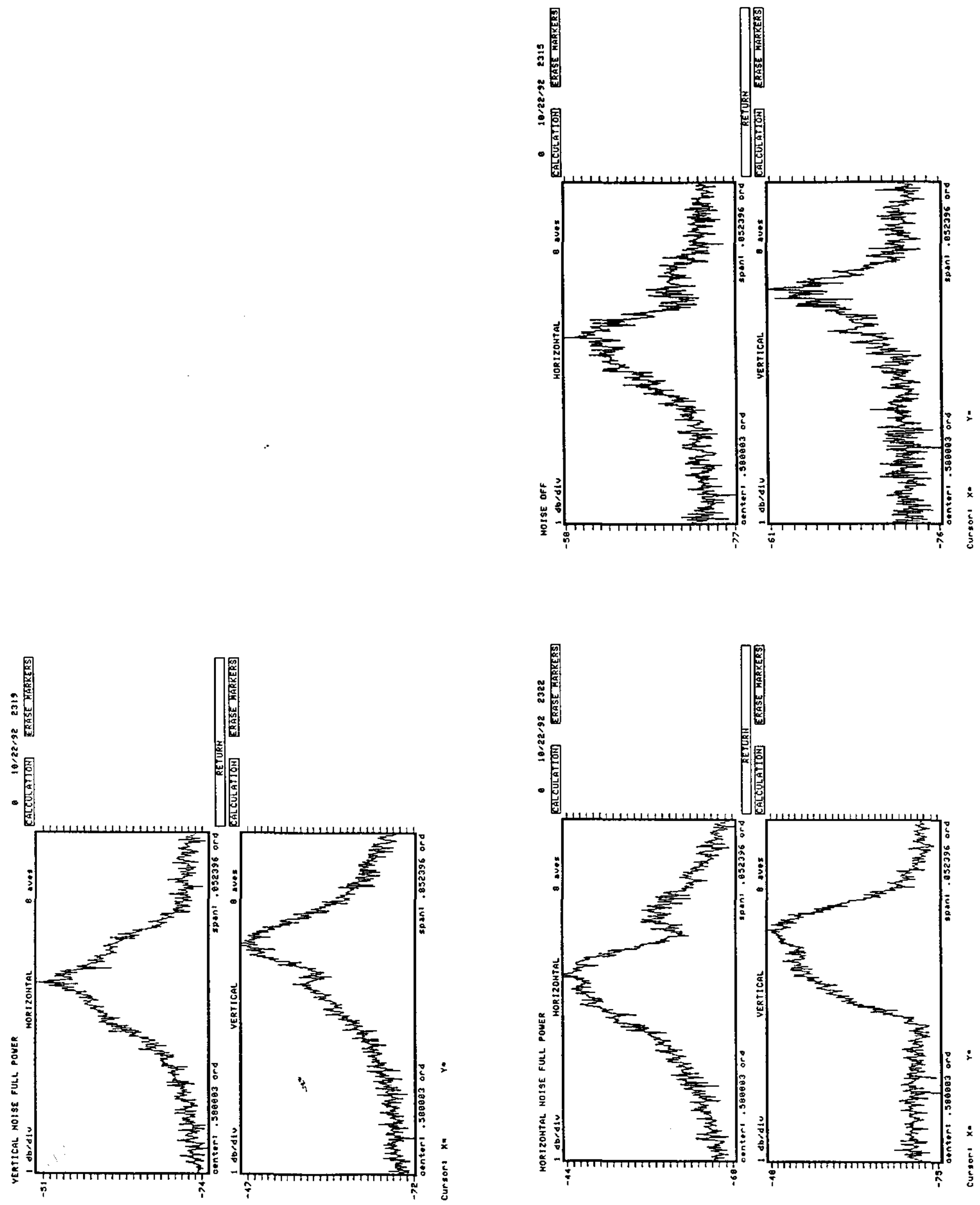